\title{
Outcomes intermedios
}

\author{
IGNACIO NEUMANN ${ }^{1,2}$, JUAN CARLOS CLARO G-A. ${ }^{1,2}$
}

\section{Surrogate outcomes}

L a comunidad médica está cada vez más consciente de la necesidad de involucrar a los pacientes crónicos en el control y manejo de su enfermedad ${ }^{1}$. Una manera de aumentar esta participación, es involucrándolos activamente en la toma de decisiones ${ }^{2}$. Al discutir las ventajas y desventajas de los distintos tratamientos, es probable que los pacientes informados tomen su decisión basados en lo que ellos esperan de dichos tratamientos o intervenciones. Vale decir, aumentar su expectativa de vida, mejorar su calidad de vida o reducir las complicaciones derivadas de su enfermedad o su tratamiento. Los outcomes relacionados al aumento de la expectativa de vida (ej. disminución de la mortalidad), a la calidad de vida (ej. infarto agudo al miocardio; necesidad de hemodiálisis) o a la reducción de complicaciones (ej. efectos adversos de medicamentos) se conocen como outcomes importantes para los pacientes.

Los outcomes intermedios (en inglés, surrogate outcomes), por otra parte, son mediciones de laboratorio o variables fisiológicas que se utilizan como sustitutos de un outcome importante para los pacientes. En la Figura 1 se muestran algunos ejemplos de esto.
'Unidad de Medicina Basada en Evidencia, Escuela de Medicina, Pontificia Universidad Católica de Chile. 2Unidad Docente Asociada, Hospital Dr. Sótero del Río, Pontificia Universidad Católica de Chile. Santiago de Chile.

Correspondencia a: Dr. Ignacio Neumann.

E-mail: ignacio.neumann@gmail.com
Los outcomes intermedios están frecuentemente presentes en la investigación. Por ejemplo, respecto de los estudios realizados en diabetes, sólo en $21 \%$ de los estudios clínicos aleatorios (ECR) publicados y en $18 \%$ de los ECR en curso se reportan outcomes considerados importantes para los pacientes ${ }^{3,4}$.

¿Qué problemas conlleva utilizar los outcomes intermedios en la toma de decisiones en salud?

Podríamos resumirlos en tres grupos:

\section{El beneficio en un outcome intermedio no se traduce necesariamente en un beneficio en un outcome importante para el paciente}

La relación entre un outcome intermedio y un outcome importante para el paciente es siempre compleja y desafía a la lógica causal. Por ejemplo, la hipertensión arterial es un factor de riesgo para el desarrollo de daño vascular, que a su vez conlleva un aumento en la incidencia de eventos cardiovasculares adversos: infarto agudo al miocardio (IAM), accidente vascular encefálico (AVE), insufi-

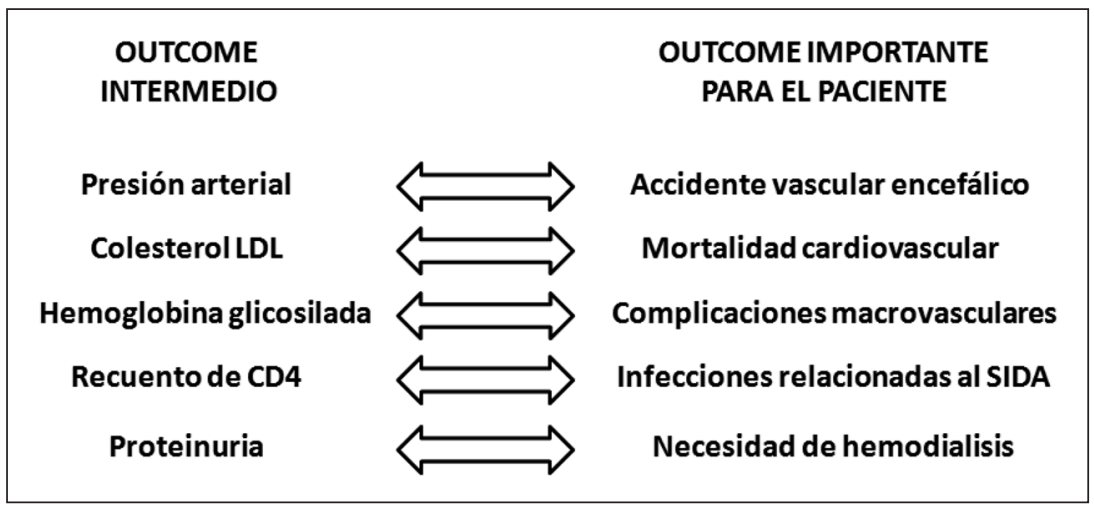

Figura 1. 
ciencia cardiaca y mortalidad cardiovascular. Existe una relación estrecha entre las cifras tensionales (outcome intermedio) y dichos outcomes (importantes para el paciente) $)^{5}$. Desde la visión fisiopatológica podemos inferir que cualquier medicamento que reduzca la presión arterial debiera tener un impacto en reducir la incidencia de AVE, IAM, falla cardiaca y finalmente en reducir la mortalidad cardiovascular. Esto es así para la mayoría de las drogas hipotensoras ${ }^{6}$, pero no parece serlo para el atenolol, que es tan eficaz como otros fármacos en reducir las cifras de presión arterial; sin embargo, no parece prevenir la incidencia de AVE ni infarto y tampoco disminuye la mortalidad cardiovascular en pacientes con hipertensión esencial ${ }^{7,8}$. Esta falta de efecto no se explica por la aparición de efectos tóxicos. El atenolol reduce la presión arterial de forma efectiva (outcome intermedio), pero no parece prevenir la aparición de outcomes importantes para los pacientes, mostrándonos que la inferencia respecto de que cualquier droga hipotensora repercute necesariamente en los outcomes importantes para los pacientes es errónea.

Otro ejemplo interesante es el efecto de los inhibidores de la enzima convertidora de angiotensina (iECA) y de los antagonistas del receptor de angiotensina tipo 2 (ARA-2) en la proteinuria y la falla renal. La proteinuria es reconocida como un factor de riesgo para el desarrollo de daño renal y de progresión de la falla renal ${ }^{9}$. Por otra parte, la asociación de iECA y ARA-2 ha demostrado reducir la proteinuria en mayor medida que cada fármaco por separado ${ }^{10}$. Nuevamente, la visión fisiopatológica nos lleva a pensar que el efecto benéfico de la asociación de iECA y ARA-2 en un outcome intermedio como la proteinuria debería traer como consecuencia una disminución en la aparición o progresión de falla renal, y finalmente de la necesidad de diálisis que es el outcome importante para el paciente. Pero dicha inferencia no parece ser correcta: en el estudio ONTARGET se observó que la terapia combinada de un iECA y un ARA-2 efectivamente reduce en mayor medida la proteinuria (outcome intermedio), pero aumenta la necesidad de diálisis (importante para el paciente) ${ }^{11}$.

\section{El beneficio en un outcome intermedio puede ocultar información acerca de posibles efectos adversos de la intervención en otros outcomes}

La capacidad de un estudio clínico para detectar diferencias significativas en las tasas de eventos depende del número de pacientes observado (tamaño de la muestra). Para detectar diferencias en eventos que ocurren con baja frecuencia, se requiere de tamaños grandes (varios miles de pacientes estudiados). Los estudios diseñados para medir outcomes intermedios son habitualmente de menor tamaño y de menor tiempo de seguimiento que los que miden outcomes importantes para los pacientes (ver explicación más abajo), por lo cual es muy improbable que estos estudios sean capaces de detectar efectos adversos de una intervención que ocurran con baja frecuencia o tras un período de tiempo prolongado. Un ejemplo de esto lo podemos encontrar en el tratamiento de la diabetes. La rosiglitazona fue aprobada en Estados Unidos de Norteamérica por la FDA (US Food and Drug Administration) en 1999 para el tratamiento de la diabetes basándose en estudios aleatorizados que probaron su beneficio en la reducción de los niveles de glicemia y de hemoglobina glicosilada ${ }^{12-14}$. Tomando únicamente la evidencia de estos estudios, la comunidad médica recibió con entusiasmo al nuevo hipoglicemiante, transformándolo en parte de los estándares de manejo de la diabetes ${ }^{15}$. A medida que se comenzó a acumular la evidencia proveniente de los estudios que evaluaban outcomes importantes para el paciente, se observó un aumento significativo de la incidencia de insuficiencia cardiaca e IAM con el uso de rosiglitazona ${ }^{16,17}$. Las agencias de control de fármacos emitieron alertas respecto de este riesgo durante el segundo semestre de 2007 y en octubre de 2008 las Sociedades Americana y Europea de Diabetes emitieron una actualización de sus recomendaciones en las cuales expresamente no se recomendaba más el uso de rosiglitazona ${ }^{18}$.

\section{Es difícil trasladar la toma de decisiones a los pacientes con argumentos basados en outcomes intermedios}

Hemos aclarado que las necesidades de los pacientes están relacionadas con aumentar la expectativa de vida, mejorar la calidad de vida o reducir las complicaciones de los tratamientos. En la medida que los outcomes intermedios no sean un fiel reflejo de estas necesidades, se convierten en una barrera para la participación del paciente en la toma de decisiones.

Por ejemplo, un paciente con hipotiroidismo subclínico puede no estar necesariamente de acuerdo con iniciar terapia de suplementación 
con levotiroxina si los beneficios que aducimos para ello son mejorar el perfil lipídico, disminuir el grosor de la íntima carotídea y mejorar otros marcadores de disfunción endotelial ${ }^{19}$.

\section{¿¿Por qué es tan frecuente la medición de outcomes intermedios en vez de outcomes importantes para los pacientes?}

Establecimos que la capacidad de un estudio clínico para detectar diferencias estadísticamente significativas en las tasas de eventos depende del número de pacientes observados. Dado que la frecuencia de eventos importantes para los pacientes (ej. mortalidad) es habitualmente baja en la mayoría de las poblaciones y que los cambios en los outcomes intermedios son relativamente frecuentes, los estudios que evalúan outcomes importantes para los pacientes requieren de mayor tamaño (más pacientes reclutados) que los que evalúan outcomes intermedios. Además, habitualmente el efecto sobre el outcome intermedio (ej. cambio en la hemoglobina glicosilada) se puede observar relativamente temprano dentro del seguimiento, a diferencia del efecto sobre el outcome importante para el paciente (ej. mortalidad). De esta forma, los estudios focalizados en outcomes intermedios requieren de seguimientos más cortos que los que miden outcomes importantes para los pacientes. El menor tamaño y un seguimiento más corto hacen a los estudios clínicos focalizados en outcomes intermedios ostensiblemente más baratos.

Otro factor importante a considerar, es la política de las agencias de medicamentos para la aprobación de fármacos. Por ejemplo, la agencia norteamericana (FDA), en áreas como la diabetes, sólo exige demostración de efectos benéficos a nivel de outcomes intermedios para autorizar la entrada de un nuevo medicamento al mercado. Esta política, en la práctica, exime a las compañías farmacéuticas de realizar estudios que evalúen outcomes importantes para el paciente.

\section{Pero, ¿podemos prescindir de los outcomes intermedios?}

Cada vez que necesitemos tomar una decisión respecto de una intervención para un paciente determinado, es ideal contar con información respecto de esta intervención en outcomes importantes para el paciente.
Sin embargo, antes de embarcarse en la costosa empresa de evaluar outcomes importantes para los pacientes, parece razonable que las nuevas intervenciones deban demostrar un beneficio significativo en outcomes intermedios. Por ejemplo, antes de probar un nuevo hipolipemiante en un gran número de personas para evaluar su efecto en la mortalidad cardiovascular, parece razonable realizar un estudio que evalúe su efecto en el colesterol LDL, ya que si no lograra demostrar un beneficio en términos del outcome intermedio (LDL), sería poco probable que demostrara un beneficio en un outcome importante para el paciente (mortalidad cardiovascular).

Sin embargo, es importante remarcar que la información que obtenemos del outcome intermedio es preliminar y, de ser favorable, debe ser corroborada por estudios que evalúen outcomes importantes para los pacientes.

\section{Conclusiones}

Los outcomes intermedios son mediciones de laboratorio o variables fisiológicas que se encuentran "a medio camino" del outcome importante para el paciente. La toma de decisiones basada en estudios que miden sólo outcomes intermedios acerca de una intervención determinada no necesariamente conlleva un beneficio real, ignora la eventual toxicidad de dicha intervención y reduce la posibilidad de participación activa por parte del paciente en la toma de decisiones. Por tanto, toda vez que esto sea posible, la toma de decisiones debiera basarse en la evidencia proveniente de estudios que evalúen outcomes importantes para los pacientes.

\section{Referencias}

1. Montori VM, Gafni A, Charles C. A shared treatment decision-making approach between patients with chronic conditions and their clinicians: the case of diabetes. Health Expect 2006; 9: 25-3.

2. Montori VM, Gandhi GY, Guyatt GH. Patient-important outcomes in diabetes-time for consensus. Lancet 2007; 370: 1104-106

3. Montori VM, Wang YG, Alonso-Coello P, Bhagra S. Systematic evaluation of the quality of randomized controlled trials in diabetes. Diabetes Care 2006; 29 (8): 1833-8. 
4. Gunjan G, Murad M, Fujiyoshi A, Mullan RJ, Flynn D, Elamin M, et al. Patient-Important Outcomes in Registered Diabetes trials. JAMA 2008; 299 (21): 2543-9.

5. Prospective Studies Collaboration. Age-specific relevance of usual blood pressure to vascular mortality: a metaanalysis of individual data for one million adults in 61 prospective studies. Lancet 2002; 360: 1903-13.

6. Blood Pressure Lowering Treatment Trialists Collaboration. Effects of different blood-pressure-lowering regimens on major cardiovascular events: results of prospectively-designed overviews of randomised trials. Lancet 2003; 362: 1527-35.

7. Carlberg B, Samuelsson O, Lindholm L. Atenolol in hypertension: is it a wise choice? Lancet 2004; 364: 1684-9.

8. Neumann I, Montero J. Análisis crítico: "[Should atenolol be the first choice for primary hypertension?]" Rev Med Chile 2005; 133 (5): 597-600.

9. Burton C, Harris K. The role of proteinuria in the progression of chronic renal failure. Am J Kidney Dis 1996; 27 (6): 765-75.

10. Kunz R, Friedrich C, Wolbers M, Mann JF. Meta-analysis: effect of monotherapy and combination therapy with inhibitors of the rennin angiotensin system on proteinuria in renal disease. Ann Intern Med 2008; 148: 30-48.

11. Mann J, Schmieder R, Mcqueen M, et al. Renal outcomes with telmisartan, ramipril, or both, in people at high vascular risk (the ONTARGET study): a multicentre, randomised, double-blind, controlled trial. Lancet 2008; 372: 547-53.

12. Nolan JJ, Jones NP, Patwardhan R, Deacon LF. Rosigli- tazone taken once daily provides effective glycaemic control in patients with Type 2 diabetes mellitus. Diabet Med 2000; 17: 287-94.

13. Patel J, Anderson RJ, Rappaport EB. Rosiglitazone monotherapy improves glycaemic control in patients with type 2 diabetes: a twelve-week, randomized, placebocontrolled study. Diabetes Obes Metab 1999; 1: 165-72.

14. Raskin P, Rendell M, Riddle MC, Dole JF, Freed MI, Rosenstock J; Rosiglitazone Clinical Trials Study Group. A randomized trial of rosiglitazone therapy in patients with inadequately controlled insulin-treated type 2 diabetes. Diabetes Care 2001; 24 (7): 1226-32.

15. Nathan D, Buse J, Davidson M. Management of Hyperglycemia in Type 2 Diabetes: A Consensus Algorithm for the Initiation and Adjustment of Therapy. Diabetes Care 2006; 29 (8): 1963-72.

16. Nissen S, Wolski K. Effect of Rosiglitazone on the Risk of Myocardial Infarction and Death from Cardiovascular Causes. N Engl J Med 2007; 356: 2457-71.

17. Singh S, Loke Y, Furberg C. Long-term Risk of Cardiovascular Events With Rosiglitazone: A Meta-analysis. JAMA 2007; 298 (10): 1189-95.

18. Nathan D, Buse J, Davidson M. Management of Hyperglycemia in Type 2 Diabetes: A Consensus Algorithm for the Initiation and Adjustment of Therapy. Diabetes Care 2008; 31: 1-11.

19. Monzani F, Caraccio N, Kozàkowà M, Dardano A, Vittone F, Virdis A, et al. Effect of levothyroxine replacement on lipid profile and intima-media thickness in subclinical hypothyroidism: a double-blind, placebo-controlled study. J Clin Endocrinol Metab 2004; 89: 2099-106. 